\title{
Belief in the American Indian/Alaska Native biological vulnerability myth and drinking to cope: Does stereotype threat play a role?
}

\section{Authors: Vivian M. Gonzalez, Adrienne Burroughs, \& Monica C. Skewes}

(C) This manuscript version is made available under the CC-BY 4.0 license.

Gonzalez, Vivian M., Adrienne Burroughs, and Monica C. Skewes. "Belief in the American Indian/ Alaska Native Biological Vulnerability Myth and Drinking to Cope: Does Stereotype Threat Play a Role?" Cultural Diversity and Ethnic Minority Psychology 27, no. 1 (January 2021): 37-46. doi:10.1037/cdp0000366. 
Belief in the Myth of an American Indian/Alaska Native Biological Vulnerability to

\begin{abstract}
Alcohol Problems Among Reservation-Dwelling Participants with a Substance Use
Problem
\end{abstract}

\author{
Vivian M. Gonzalez ${ }^{1}$ and Monica C. Skewes ${ }^{2}$ \\ ${ }^{1}$ Department of Psychology, University of Alaska Anchorage \\ ${ }^{2}$ Department of Psychology, Montana State University
}

\begin{abstract}
Author Note
This research was supported by the National Institute of General Medical Sciences of the National Institutes of Health under Award Number 5P20GM104417-02. The content is solely the responsibility of the authors and does not necessarily represent the official views of the National Institutes of Health. The authors express sincere gratitude to the Community Advisory Board for their guidance and feedback on this project, and to the participants who generously shared their time and experiences. Correspondence concerning this article should be addressed to Monica C. Skewes, Montana State University, Department of Psychology, 320 Traphagen Hall, Bozeman, Montana 59717. Email: monica.skewes@montana.edu.
\end{abstract}




\begin{abstract}
Background: Belief in the myth of an American Indian/Alaska Native (AIAN) specific biological vulnerability (BV) to alcohol problems is associated with worse alcohol outcomes among AIAN college students who drink, despite also being associated with greater attempts to reduce drinking. This study examined the association of belief in a BV with alcohol use among reservation-dwelling AI adults with a substance use problem. Methods: Participants $(n=141)$ who drank alcohol in the past 90 days were selected from a larger AI sample who self-identified as having a substance use problem. Moderated-mediation analyses examined whether belief in a
\end{abstract} BV was positively associated with alcohol use and substance use consequences, as well as whether self-efficacy and craving mediated the association of belief in a BV with alcohol use. Results: Among participants who reported using alcohol but not hard drugs (e.g., methamphetamine, opioids), greater belief in a BV was associated with greater drinking days, which in turn was associated with greater consequences. Among participants who used alcohol only, belief in a BV was also significantly associated with greater craving, and in turn with greater drinking days. Among those who used both alcohol and hard drugs, greater belief in a BV was associated with fewer drinking days, but was not significantly associated with consequences. No association was found between belief in a BV and self-efficacy to avoid alcohol or drug use. Conclusions: Among those who use only alcohol, belief in a BV may contribute to greater drinking days and consequences through its association with greater craving. This study provides further evidence of the potential harm of internalizing the belief that being AIAN contributes to risk for alcohol problems, a notion that lacks scientific evidence despite decades of research. The findings highlight the importance of combatting societal myths regarding AIAN peoples and the internalization of these stereotypes.

Keywords: American Indian, alcohol, stereotype, biological vulnerability, firewater myth 


\section{Introduction}

American Indians and Alaska Natives (AIANs) have higher rates of current and lifetime abstinence from alcohol compared with non-AIANs in the U.S. (Beals et al., 2003; Cunningham et al., 2016). However, among those who drink there is evidence of greater alcohol consumption and alcohol use disorder (AUD; Beals et al., 2003; Vaeth et al., 2017). There also is evidence of greater alcohol-related morbidity and mortality, and greater severity of AUD for AIANs than for non-AIANs (Grant et al., 2015; Vaeth et al., 2017; Whitesell et al., 2012).

There are a number of factors contributing to alcohol-related health disparities for AIANs. These include inequities in the social determinants of health, such as greater poverty and unemployment, lower attainment of formal education, greater exposure to trauma, and more limited access to health care (Brave Heart et al., 2016; Castor et al., 2006; Collins, 2016; Vaeth et al., 2017; Whitesell et al., 2012). Other contributing factors include the negative effects of discrimination on health and the lasting effects of historical trauma that are associated with the violent colonization and forced assimilation of AIANs by European descendants (Cheadle \& Whitbeck, 2011; Whitbeck et al., 2001, 2004). In a national epidemiological dataset, Brave Heart et al. (2016) found that when socioeconomic variables were adjusted for, differences in the rates of AUD between AIANs and non-Hispanic Whites were no longer significant, suggesting that socioeconomic variables largely account for observed racial differences.

Based on the high lifetime rate of AUD in the U.S. (29.1\%), many people struggle with moderating their drinking and experience impairing alcohol consequences (Grant et al., 2015). Controlling for demographics (e.g., age, marital status, income, and education), the lifetime rate of AUD among White Americans is at least double that of Hispanics, African Americans, and Asian Americans (Grant et al., 2015), a difference that does not appear to be attributed to race by 
scientists or the general population. In contrast, AIANs are often subjected to biological and genetic explanations for alcohol-related health disparities (Skewes \& Lewis, 2016) and these explanations also have been applied to Indigenous peoples in Canada (Johnson, 2016; Thatcher, 2004). Despite a number of studies attempting to identify biological markers of risk for AUD among AIANs, there is a notable lack of evidence to support the notion that biological or genetic factors play a greater role in AUD among AIANs compared to other racial groups (Ehlers \& Gizer, 2013; Enoch \& Albaugh, 2017). However, many people still believe that there is an AIAN specific biological vulnerability (BV) to alcohol problems (Gonzalez \& Skewes, 2018).

While biogenetic explanations for mental health issues, such as alcohol and other substance use disorders, have been thought to hold promise for reducing the stigma of these disorders, research suggests that attributing mental health problems to biogenetic causes does not reduce public stigma (Angermeyer et al., 2011). For example, between 1996 and 2006, while genetic or chemical imbalance causal attributions increased for AUD in the U.S., there was no reduction in social stigma. Rather, there was an increase in attributions regarding sufferers" "bad character" as causal in AUD (Pescosolido et al., 2010). One potential problem with biogenetic explanations is that they may contribute to the misperception that mental health issues are incurable (Andersson \& Harkness, 2018).

Placing causal explanations for alcohol-related health disparities in racially-based biogenetic differences may negatively affect AIANs who choose to drink. Such causal attributions also may increase racial bias and discrimination against AIAN peoples, regardless of drinking behavior (LaMarr, 2003). For AIANs who are not abstinent, the implicit message is that they drink at their own peril, which may negatively affect attempts to moderate drinking (Gonzalez \& Skewes, 2016). Thus, an internalized belief in a BV may contribute to the future 
fulfillment of that expectation among those who are not lifetime abstainers (LaMarr, 2003).

\section{BV Belief and Alcohol Outcomes in College Drinkers}

To date, research on belief in a BV and alcohol outcomes among AIANs who drink has been conducted only with college students, and findings suggest that this belief is associated with harmful outcomes (Gonzalez et al., 2019, 2021; Gonzalez \& Skewes, 2016, 2018). For example, belief in a BV is associated with greater depression and drinking to cope (Gonzalez et al., 2021); lower self-efficacy to avoid drinking heavily; greater heavy episodic drinking and temptation to drink heavily; and greater negative alcohol consequences, despite also being associated with guilt for drinking even small amounts of alcohol and greater attempts to control drinking (Gonzalez \& Skewes, 2016). In addition to potentially influencing affect and cognition about alcohol as well as self-efficacy to control intake, this belief also may contribute to greater drinking and consequences through its effect on strategies used to avoid alcohol consequences. Among AIAN students who drink, greater BV belief was associated with greater use of abstinence-based strategies, which were ineffective or even counterproductive for avoiding alcohol consequences (Gonzalez \& Skewes, 2018), less use of effective strategies that minimize consequences (i.e., protective behavioral strategies; Gonzalez et al., 2019), and lower selfefficacy to use harm reduction strategies (Gonzalez et al., 2019).

\section{Potential Influence of BV Belief on Recovery}

Studies with college students suggest that for AIANs who drink, there may be negative psychological and behavioral health ramifications of believing in the myth of an AIAN-specific $\mathrm{BV}$, which may make this belief a risk factor for developing problematic alcohol use (Gonzalez et al., 2019, 2021; Gonzalez \& Skewes, 2016, 2018). This myth also may negatively affect AIAN individuals who are trying to overcome alcohol problems (Gonzalez \& Skewes, 2016). To 
date, no studies have examined belief in a BV with AIAN community, clinical, or recovery samples.

Given the association of belief in a BV with lower self-efficacy in college students who drink (Gonzalez \& Skewes, 2016), this belief may likewise impact self-efficacy among those in recovery. Self-efficacy, or confidence in one's ability to avoid substance use in tempting situations, is an important predictor of treatment success, including reduced substance use (Kadden \& Litt, 2011) and longer time to relapse (Greenfield et al., 2000). Belief in a BV may lead those experiencing substance use problems to feel that their use is due to biogenetic factors that are out of their control, negatively impacting self-efficacy to avoid substance use.

Craving (i.e., temptation or urges to use substances and experiencing these as difficult to control) is another variable that robustly predicts treatment outcomes in clinical populations and may be affected by belief in a BV. Studies show that greater alcohol craving prospectively predicts treatment dropout among people with AUD (O'Connor et al., 1991) as well as relapse following treatment (Chong \& Lopez, 2008; Gordon et al., 2006; Stohs et al., 2019). In previous research with college students, belief in a BV was positively associated with greater temptation to drink (Gonzalez \& Skewes, 2016); belief in a BV may be similarly associated with greater craving and alcohol use among those experiencing more serious substance use issues.

\section{Current Study}

In this study, we examined the associations of belief in a BV with alcohol use and substance use consequences among reservation-dwelling AI adults who self-identified as having a substance use problem. First, we examined whether belief in a BV was associated with alcohol use (i.e., number of drinking days and drinks per drinking day) and whether alcohol use in turn was associated with greater substance use consequences. This study then focused on 
understanding how BV belief may impact alcohol use through its association with self-efficacy and craving. We hypothesized that greater belief in a BV would be associated with greater craving, which in turn would be associated with greater alcohol use. We further hypothesized that greater belief in a BV would be associated with lower self-efficacy to avoid substance use in tempting situations, which in turn would be associated with greater alcohol use.

Hypotheses for the study were based on research and theory for people who use alcohol, and it was unknown how using hard drugs (e.g., methamphetamine, opioids) in addition to drinking might change the nature of the associations. Therefore, moderation by substance use group (alcohol only vs. both alcohol and hard drugs) was examined to avoid a potential aggregation error in which one fails to identify subgroups that may differ, leading to errors in inference (Osborne, 2019); these comparisons were exploratory in nature.

\section{Method}

\section{Participants}

The data for the present study came from a survey administered as part of an ongoing community based participatory research (CBPR) project that aimed to understand substance use and develop culturally grounded intervention strategies for tribal members from a rural AI reservation (Skewes et al., 2020). Participants in the larger survey study were 198 AI adults from the reservation where the research took place who self-identified as "having a substance use problem and trying to recover."

Given the focus of the current study on the associations of belief in a BV with alcohol use and substance use consequences, only individuals who reported having at least one drink in the 90 days prior to the survey were included in analyses examining alcohol use $(n=141)$. Seven participants in the larger survey who were missing substance use assessments due to researcher 
error were excluded from all analyses.

Among participants who reported alcohol use, 47.5\% were women and 52.5\% were men, with a mean age of 38.0 years (see Table 1 for other participant characteristics). Participants were abstinent from alcohol the majority of days, with a mean of $70.5 \%$ days abstinent in the 90 day assessment period $(S D=31.4, M d=86.7)$. In this sample, $40.4 \%(n=57)$ used "hard drugs" (i.e., drugs other than cannabis). Among those who used hard drugs, the most commonly used was methamphetamine $(96.5 \%, n=55)$ with a small minority reporting opioid use $(12.3 \%, n=7)$. Participants who used hard drugs were abstinent the majority of days, with a mean of $67.5 \%$ of days abstinent from hard drugs $(S D=29.3, M d=74.4)$.

\section{Procedure}

This project represents a long-term collaboration between academic and community research partners from a reservation in the Northern Plains region of the U.S. Using a CBPR framework (Israel et al., 2008; Wallerstein \& Duran, 2010, 2016), we sought input from a community advisory board (CAB) of AI community members and a local project manager who were instrumental in the project. The $\mathrm{CAB}$ and the tribal IRB approved the study, including the procedures, measures, and this manuscript.

Recruitment began when the local project manager made an in-person announcement to a group of eight patients at a recovery support group at the on-reservation outpatient treatment center. The project manager described the history of the project and the CBPR approach, gave a summary of findings from the formative qualitative phases of the project (see Skewes \& Blume, 2019; Skewes et al., 2019, 2020), and extended an invitation to contact the research team at the local tribal college if interested in participating. After this initial recruitment effort, news of the survey spread rapidly across the reservation, with subsequent recruitment taking place 
exclusively through word of mouth.

Data were collected during in-person one-on-one interviews conducted by a member of the research team, which included the non-AI academic partners, the AI project manager, and two AI members of the CAB. Following an introduction to the larger project and informed consent, participants completed interview and self-report measures. For participants who reported problems with reading, all items were read aloud by the interviewer. After the survey was complete, participants received a referral sheet with contact information for local substance use disorder (SUD) and other mental health treatment, and were thanked with a $\$ 50$ gift card.

\section{Measures}

Substance use. Substance use in the previous 90 days was assessed with the Timeline Followback interview (TLFB; Sobell \& Sobell, 1992). The TLFB is a widely used calendarbased daily recall method that yields valid and reliable estimates of alcohol and drug consumption (Robinson et al., 2014; Sobell et al., 1992, 2003). Variables calculated from TLFB data included number of drinking days and number of standard drinks per drinking day (total standard drinks/drinking days) in the 90-day assessment period. Standard drinks were defined as $12 \mathrm{oz}$. of beer, $5 \mathrm{oz}$. of wine, 8 to $9 \mathrm{oz}$. of malt liquor, or $1.5 \mathrm{oz}$. of 80 -proof liquor.

Substance use consequences. An adapted version of the Drinker Inventory of Consequences (DrInC; Miller et al., 1995) was used to assess alcohol and drug consequences in the month prior to assessment. This adaption was based on the Drinker Inventory of Consequences for Alaska Natives (DrInC-AN; Allen, 2007), which is itself a modified version of the DrInC (Miller et al., 1995) that was designed to increase cultural sensitivity and relevance for Indigenous participants. The DrInC-AN includes at least minor linguistic or cultural modifications for the majority of DrInC items (e.g., including giving away too much money to 
the consequence of losing or spending too much money) as well as items that were added (e.g., having been disrespectful of people as a consequence). In the current study, the 50 item DrInCAN was further modified to refer to alcohol or drug use, making the scale similar to the Inventory of Drug Use Consequences (InDUC-2R; Miller et al., 1995) which has established test-retest reliability and validity with individuals who use substances (Blanchard et al., 2003; Tonigan \& Miller, 2002). For this study, items were rated as present (1) or absent (0) in the past 30 days and summed to yield a total score. Internal consistency in the present sample was high, with a Cronbach's alpha of .98.

Craving. Craving was assessed using a modified version of the Penn Alcohol Craving Scale (PACS; Flannery et al., 1999). The PACS is a valid and reliable measure that includes five items assessing subjective experiences of craving alcohol and difficulty controlling urges in the past week (Flannery et al., 1999). In the present study, items were modified to assess craving for alcohol or drugs. Items were rated on a scale from 1 to 7 , with unique response options for each item. Responses were summed to yield a total score, with higher scores indicating greater craving. Internal consistency was high in the present sample, with a Cronbach's alpha of .92.

Self-efficacy. Self-efficacy was assessed using a modified version of the 8-item Brief Situational Confidence Questionnaire (BSCQ; Breslin et al., 2000), an instrument with good psychometric properties that was derived from a longer version (Annis \& Graham, 1988; Breslin et al., 2000). The BSCQ assesses confidence in one's ability to avoid drinking heavily across a variety of tempting situations. In the present study, the scale was modified to refer to ability to resist drinking heavily or using their drug of choice. Each item was rated on a scale from $0 \%$ (not at all confident) to $100 \%$ (totally confident). A mean score was calculated to yield an overall self-efficacy score. Internal consistency was high in the present sample (Cronbach's alpha $=.89)$. 
Belief in a biological vulnerability. Belief in an AIAN BV to alcohol problems was assessed using three items from the 9-item BV subscale of the Revised Firewater Myth Scale (RFMS; Gonzalez \& Skewes, 2016). These items were selected because they had the strongest factor loadings on the subscale (Gonzalez \& Skewes, 2016) and were central to the construct of a BV. Items included the following: “Alaska Natives and American Indians feel the effects of alcohol, or feel intoxicated, more easily than people of European descent," "Alaska Natives and American Indians metabolize alcohol differently than non-Native people," and "Alaska Natives and American Indians are more likely to have a genetic vulnerability to problems with alcohol." Items were rated from 1 (strongly agree) to 6 (strongly disagree), then reversed scored. A mean score was calculated, with higher scores indicating greater belief in a BV. In this sample, internal consistency was high (Cronbach's alpha=.89) as were inter-item correlations (.74 to .78$)$.

\section{Analyses}

Prior to analyses, data were screened following the procedures outlined in Tabachnick and Fidell (2014). Six participants were extreme univariate outliers $(z>3.29)$ on drinks per drinking day. Outlying scores were changed to be a unit higher than the next most extreme score in the distribution, thereby reducing the score's influence while maintaining its position in the distribution of scores (Tabachnick \& Fidell, 2014). Drinking days, drinks per drinking day, and substance use consequences were positively skewed and were square-root transformed prior to analyses. Missing data were minimal $(<2 \%)$. Missing scores were imputed using expectation maximization in SPSS 25 for three cases missing consequence scores and one case missing the PACS.

Moderated-mediation analyses were conducted using bias-corrected bootstrap resampling (10,000 bootstrap samples) to test the significance of the indirect effects using the Hayes 
PROCESS macro (version 3.5; Hayes, 2018). Statistical significance of the indirect effects was determined by $95 \%$ bias-corrected unstandardized bootstrapped confidence intervals that did not contain zero. Separate analyses were conducted to examine whether greater belief in a BV was associated with (a) more drinking days and (b) more drinks per drinking day ( $a$ paths in separate models), and whether these alcohol use variables in turn were associated with greater substance use consequences ( $b$ paths). Next, moderated-mediation models were used to examine whether self-efficacy and craving mediated the association of belief in a BV with alcohol use. Four separate models were conducted to examine associations between each potential mediator (selfefficacy and craving) and alcohol use variables (drinking days and drinks per drinking day). Potential differences in the nature of the associations between those who used alcohol only (AO; $n=84$; coded 0 ) and those who used both alcohol and hard drugs (HD; $n=57$; coded 1 ) were examined for all paths of the mediation models. Continuous variables that formed interaction terms were mean centered to avoid multicollinearity. Gender and age were included as covariates in all models.

\section{Results}

Means, standard deviations, ranges, and intercorrelations of the study variables are presented in Table 2. Mean drinking days in the past 90 days was $26.57(S D=28.26 ; M d=12)$. Drinks per drinking day on average was $17.97(S D=13.16 ; M d=14)$. Mean belief in a BV was $3.86(S D=1.58$; median and mode $=4.00)$, with $59.6 \%(n=84)$ endorsing some degree of belief in a BV with a mean score of "slight agreement" (rating of 4) or higher. There was no significant difference between substance use groups in belief in a BV or in alcohol use (see Table 2).

\section{Association of Belief in a BV with Abstinence}

To examine whether belief in a BV covaried with abstinence, as alcohol abstainers were 
excluded from analyses examining the association of belief in a BV with alcohol use, multiple regression analyses were conducted with the full sample who completed the TLFB $(n=191)$, controlling for age and gender. No difference in belief in a BV was found between those who were abstinent from alcohol (regardless of drug use; $n=50)$ and those who drank $(n=141$; $\mathrm{B}[\mathrm{SEB}]=-.15[.26], \beta=-.04, p=.559, f^{2}=.00$ ). Likewise, abstainers from all substances (alcohol, hard drugs, cannabis; $n=24)$ compared with non-abstainers $(n=167)$ did not differ in belief in a $\mathrm{BV}\left(\mathrm{B}[\mathrm{SEB}]=-.03[.34], \beta=-.01, p=.918, f^{2}=.00\right)$.

\section{Belief in a BV $\rightarrow$ Alcohol Use $\rightarrow$ Substance Use Consequences}

Drinking days. In the $a$ path of the model examining drinking days as a mediator of the association between belief in a BV and substance use consequences, belief in a BV was significantly associated with drinking days (see Table 3 and Figure 1), but this relationship was moderated by substance use group. In the AO group, as belief in a BV increased so did number of drinking days $\left(\mathrm{B}[S E]=0.42[0.17], \beta=.25, p=.015, f^{2}=.05\right)$. In contrast, for the HD group, greater belief in a $\mathrm{BV}$ was associated with fewer drinking days $(\mathrm{B}[S E]=-0.44[0.21], \beta=-.26$, $p=.038, f^{2}=.03$; see Figure 2).

In the $b$ path of the model, participants with greater drinking days experienced significantly greater consequences and substance use groups did not differ in this association. However, the HD group had significantly greater substance use consequences than the AO group. Examining the model $c^{\prime}$ path, there was not a significant direct association of belief in a $\mathrm{BV}$ with substance use consequences for either group. For the $\mathrm{AO}$ group there was a significant indirect association between belief in a BV and substance use consequences through BV's positive association with drinking days $(\mathrm{B}[S E]=0.10[0.05], 95 \% \mathrm{CI}[0.01,0.20])$, while for the HD group no indirect association was found $(\mathrm{B}[S E]=-0.13[0.10], 95 \% \mathrm{CI}[-0.35,0.01])$. 
Drinks per drinking day. In the model examining drinks per drinking day as a mediator of the association of belief in a BV with substance use consequences, belief in a BV and its interaction with substance use group were not significantly associated with drinks per drinking day, nor was substance use group (see Table 3).

Examining the $b$ path of the model, drinks per drinking day and the interaction of drinks per drinking day by substance group were not significantly associated with consequences. Further, there was no direct association between belief in a BV and substance use consequences for either group, nor was there a significant indirect effect for either the $\mathrm{AO}(\mathrm{B}[S E]=0.00[0.03]$, 95\% CI[-0.07, 0.04]) or HD group (B[SE]=-0.01 [0.03], 95\% CI[-0.09, 0.05]).

\section{Belief in a BV $\rightarrow$ Self-Efficacy $\rightarrow$ Alcohol Use}

Drinking days. In the model examining self-efficacy as a mediator of the association of belief in a BV with drinking days, belief in a BV and its interaction with substance use group were not significantly associated with self-efficacy (see Table 4). The HD group had significantly lower self-efficacy than the AO group.

Examining the $b$ path of the model, lower self-efficacy was significantly associated with more drinking days. Substance use groups did not differ in the association between self-efficacy and drinking days. Belief in a BV was directly associated with drinking days, but substance use groups differed significantly in the nature of this association. There was a significant direct association of belief in a $\mathrm{BV}$ with greater drinking days for the $\mathrm{AO}$ group $(\mathrm{B}[S E]=.45[.16]$, $\beta=.27, p=.006, f^{2}=.06$ ); however, belief in a BV was negatively associated with drinking days for the HD group $\left(\mathrm{B}[S E]=-0.40[.20], \beta=-.24, p=.046, f^{2}=.03\right)$. The indirect effect was not significant for either the $\mathrm{AO}(\mathrm{B}[S E]=-0.03[0.07], 95 \% \mathrm{CI}[-0.22,0.08])$ or HD group $(\mathrm{B}[S E]-0.04[0.08]$, $95 \%$ CI[-0.19, 0.14]). 
Drinks per drinking day. For this model, the association of belief in a BV with selfefficacy was identical to that reported for the drinking days model (see Table 4). Self-efficacy and substance use group were not significantly associated with drinks per drinking day. There was no direct effect of belief in a BV with drinks per drinking day and substance use groups did not differ significantly in this association. The indirect effect of belief in a BV on drinks per drinking day through self-efficacy was not significant for either the $\mathrm{AO}(\mathrm{B}[S E]=-0.004[0.02]$, $95 \% \mathrm{CI}[-0.04,0.03])$ or HD group $(\mathrm{B}[S E]=-0.002[0.02], 95 \% \mathrm{CI}[-0.04,0.06])$.

\section{Belief in a BV $\rightarrow$ Craving $\rightarrow$ Alcohol Use}

Drinking days. Greater belief in a BV was associated with higher reported craving for alcohol and/or drugs (see Table 5 and Figure 1). The HD group reported significantly greater craving. The interaction of belief in a BV with substance use group was not significant, suggesting that the positive association of belief in a BV with greater craving did not differ by substance use group. Examining the $b$ path of the model, greater craving was significantly associated with a greater number of drinking days, with no significant difference found between substance use groups in the effect of craving on drinking days.

For the AO group, there was a significant indirect association between belief in a BV and drinking days through BV's positive association with craving $(\mathrm{B}[S E]=0.11[0.07], 95 \% \mathrm{CI}[0.01$, $0.26])$. For the HD group, no indirect association was found $(\mathrm{B}[S E]=0.03[0.09], 95 \% \mathrm{CI}[-0.15$, 0.22). Moderation by substance use group was also found for the direct effect of BV to drinking days, with no significant association found for the AO group (B $[S E]=0.32[0.17], \beta=.19$, $p=.063, f^{2}=.03$ ); however, as in previous models for the HD group, a significant negative association was found $\left(\mathrm{B}[S E]=-0.45[0.20], \beta=-.28, p=.021, f^{2}=.04\right)$.

Drinks per drinking day. Craving was not significantly associated with drinks per 
drinking day (see Table 5). There was no direct effect of belief in a BV with drinks per drinking day, and substance use groups did not differ significantly in this association. The indirect effect of belief in a BV on drinks per drinking day through craving was not significant for either the $\mathrm{AO}(\mathrm{B}[S E]=-0.00[.02], 95 \% \mathrm{CI}[-0.05,0.05])$ or the HD group $(\mathrm{B}[S E]=-0.001[0.03], 95 \% \mathrm{CI}[-$ $0.05,0.08])$.

\section{Discussion}

To date, studies examining the association of belief in an AIAN specific BV to alcohol problems have focused on college students who drink. These studies have found either direct associations of belief in a BV with greater alcohol use and consequences (Gonzalez \& Skewes, 2016) or indirect associations of belief in a BV with these alcohol outcomes through less use of effective protective behavioral strategies (Gonzalez et al., 2019). This study sought to expand this research by examining the associations of belief in a BV with alcohol use and substance use consequences among reservation-dwelling AIs who self-identified as having a substance use problem, most of whom also reported being in recovery. We found a relatively high rate of belief in the notion of an AIAN specific BV in this sample (60\%), similar to the rate found in AIAN college student drinkers (53\%; Gonzalez \& Skewes, 2018). We also found positive associations between belief in a BV, alcohol use, and substance use consequences in this sample, although these associations depended on whether participants used alcohol only or used both alcohol and hard drugs.

Among participants who used alcohol only, belief in a BV had a small association with greater drinking days in the past 90 days, which in turn was associated with greater past month substance use consequences. Among those who used both alcohol and hard drugs, greater belief in a BV was associated with fewer drinking days but there was not a significant indirect 
association of belief in a BV with reduced substance use consequences. While this potentially could be considered a positive effect of belief in a BV, participants who used hard drugs had worse outcomes including lower self-efficacy, greater craving, and worse substance use consequences. Thus, any benefit in this regard would appear to be insubstantial.

This study also explored potential mechanisms by which belief in a BV may affect alcohol use. In our prior studies with college students who drink, greater belief in a BV was associated with lower self-efficacy for avoiding heavy drinking in tempting situations (Gonzalez \& Skewes, 2016) as well as lower self-efficacy for using harm reduction strategies (Gonzalez \& Skewes, 2018). However, in the current study belief in a BV was not significantly associated with participants' self-efficacy to resist drinking heavily or using their drug of choice in tempting situations. It may be that among participants in this sample, who were older and had more serious substance use problems compared with college students, self-efficacy was more influenced by their substantial personal struggles with substance use, such as unsuccessful attempts to quit or moderate their use and a disheartening accumulation of consequences despite change efforts. Consistent with other studies that have found a positive influence of self-efficacy on outcomes (Chong \& Lopez, 2008; Greenfield et al., 2000; Kadden \& Litt, 2011), in this study greater self-efficacy was associated with fewer drinking days for both substance use groups.

While no association was found between belief in a BV and self-efficacy in this study, greater belief in a BV had a small association with greater substance use craving, which previous studies have shown has a negative influence on recovery efforts following treatment in primarily White samples (Gordon et al., 2006; Stohs et al., 2019) as well as among AI women (Chong \& Lopez, 2008). No difference was found between groups in the positive association of belief in a BV with craving or the association of greater craving with greater drinking days. However, in the 
HD group there was no indirect effect from belief in a BV to greater drinking days through substance use craving, while in the $\mathrm{AO}$ group greater belief in a $\mathrm{BV}$ was indirectly associated with greater drinking days through its association with greater craving. This is consistent with findings with AIAN college students who drink, where greater belief in a BV was associated with greater temptation to drink heavily despite also being associated with efforts to reduce drinking (Gonzalez \& Skewes, 2016). Believing that struggles to control alcohol use are ingrained or "hard wired" for AIANs by biogenetics may affect how alcohol craving is experienced. Craving may be experienced as more intense (stronger subjective urges) or as more difficult to control when BV belief is high. In fact, the notion of AIAN people having particular difficulty controlling their drinking is a stereotype that is related to the notion of a BV (LaMarr, 2003). Given the cross-sectional nature of the study, it is important to consider the possibility that individuals who experience stronger craving may interpret this as evidence that there is a BV for AIAN people.

In this study, drinks per drinking day was used as a measure of intensity of drinking. In contrast to our findings that greater belief in a BV was associated with drinking days, there were no associations found between belief in a BV and drinks per drinking day. There also was no association of drinks per drinking day with substance use consequences. In fact, with the exception of women drinking significantly fewer drinks compared with men, this variable was not correlated with any other study variable. This was contrary to our expectation and may indicate issues with the validity of drinks per drinking day in this sample as measured or as calculated by the TLFB. In a prior study comparing the accuracy of TLFB drinking estimates relative to a smartphone-based daily diary record of drinking among individuals with an AUD (Dulin et al., 2017), the TLFB retrospective recall of drinks per drinking day tended to diverge 
more from participants' daily diary recordings of drinking than did frequency estimates. The discrepancy between the two measures increased quickly the further back participants were asked to remember (i.e., declines within two weeks of retrospective recall). This suggests that recalling drinks per drinking day is more difficult than recalling the frequency of drinking days.

\section{Limitations and Future Directions}

The primary limitation of this study is its cross-sectional design. Although we examined indirect effects using mediation analyses, there are significant limitations to this approach with cross-sectional data and causal inferences cannot be made regarding the temporal order of constructs (O'Laughlin et al., 2016). To date, no study has examined change in BV belief over time. It is possible that, barring a direct intervention or presentation of contrary evidence, this belief would be static, making it difficult to study as a causal variable. Future research is needed to examine the stability of BV belief, how change in this belief may be related to changes in alcohol outcomes or factors such as self-efficacy and craving, and how malleable this belief is with intervention efforts to debunk it.

Other limitations include the use of multiple tests and the possibility of Type I error inflation, as well as a sample size that may have limited power to detect small effects. Additionally, it is important to note that lifetime abstainers were not included in this study. While it is possible that belief in a BV may be protective for some individuals who abstain due to alcohol-related stereotypes and awareness of the role of alcohol in the colonization and oppression of AIAN peoples (Daisy et al., 1998), we did not find an association between belief in a BV and abstinence in the larger sample. Future studies are needed to understand whether belief in the BV myth is protective for some individuals or under certain circumstances. The evidence to date suggests that this belief is associated with negative outcomes. 
Another limitation is that this study used a word-of-mouth strategy to recruit participants who self-identified as having a problem with substances and trying to recover. While many participants reported "trying to recover" during the interview, definitions of recovery vary, as do definitions of having a "substance use problem." It is unknown if belief in a BV is associated with recovery efforts among those in treatment, or if these study results would generalize to those diagnosed with a SUD.

The grand majority of participants in the HD group were using methamphetamine, thus results may not generalize to other types of hard drugs use (e.g., opioids). Further, participants were grouped into only two categories given the sample size, but drug use varied within these groups, including cannabis use in both groups and varied drug use in the HD group. It also is important to note that assessments of consequences, self-efficacy, and craving referred to alcohol and/or drugs rather than to each substance separately. In the AO group, participant ratings were more specific to alcohol, whereas ratings were influenced by multiple substances in the HD group. This may have obscured some associations, or in the case of the findings for craving, may indicate that belief in a BV is associated with an increase in alcohol and/or drug use craving. Future studies are needed to examine alcohol-specific measures with individuals who use both alcohol and drugs. In addition, future studies are needed to examine whether belief in a BV is uniquely associated with the use of drugs other than alcohol. Finally, this study used quantitative methods to examine how belief in a BV is associated with substance use outcomes. Qualitative research exploring individuals' experiences, attributions, and beliefs may help to shed more light on the nature of the associations or put these findings into context, elucidating how this belief may affect experiences and outcomes. 


\section{Conclusion}

Many Americans struggle with alcohol use and problems (Grant et al., 2015). Attributing these struggles to race is inaccurate, unhelpful for change efforts, and may lead to discrimination as well as potentially misguided prevention or intervention efforts. These could inadvertently contribute to problems rather than helping to relieve them-for example, by promoting the notion that AIANs cannot drink alcohol in any amount without developing an AUD, or that harm reduction interventions that are effective with many problematic drinkers would be ineffective for AIANs. Focusing on immutable biological risk characteristics also distracts from efforts to address the social determinants of health known to contribute to alcohol problems, such as poverty, trauma, discrimination, and more limited access to healthcare.

The current study was the first to examine belief in a BV with an AI reservation community sample and focused on individuals who self-identified as having a substance use problem. In the current sample, similar results were found to our previous work with college students who drink and provides further evidence of the potential harm incurred by internalizing the belief that being AIAN contributes to a vulnerability to alcohol problems due to biology or genetics, which may become a self-fulfilling prophecy (LaMarr, 2003). It is important to combat not only internalized stereotypes, but larger societal myths of AIAN peoples' unique biogenetic vulnerability to alcohol problems. While this study found that belief in a BV was associated with greater craving and alcohol use among the AO group, future research is needed to examine whether debunking the myth of an AIAN BV during substance use interventions helps to improve outcomes. 


\section{References}

Allen, J. (2007). The Drinker Inventory of Consequences for Alaska Natives (DrInC-AN): An instrument for assessing adverse consequences of alcohol abuse preliminary manual. Unpublished manuscript.

Andersson, M. A., \& Harkness, S. K. (2018). When do biological attributions of mental illness reduce stigma? using qualitative comparative analysis to contextualize attributions. Society and Mental Health, 8, 175-194. https://doi.org/10.1177/2156869317733514

Angermeyer, M. C., Holzinger, A., Carta, M. G., \& Schomerus, G. (2011). Biogenetic explanations and public acceptance of mental illness: Systematic review of population studies. British Journal of Psychiatry, 199, 367-372.

https://doi.org/10.1192/bjp.bp.110.085563

Annis, H. M., \& Graham, J. M. (1988). Situational confidence questionnaire (SCQ-39) user's guide. Toronto: Alcoholism and Drug Addiction Research Foundation.

Beals, J., Spicer, P., Mitchell, C. M., Novins, D. K., Manson, S. M., Big Crow, C. K., Buchwald, D., Chambers, B., Christensen, M. L., Dillard, D. A., DuBray, K., Espinoza, P. A., Fleming, C. M., Frederick, A. W., Gurley, D., Jervis, L. L., Jim, S. M., Kaufman, C. E., Keane, E. M., Klein, S. A., Lee, D., McNulty, M. C., Middlebrook, D. L., Moore, L. A., Nez, T. D., Norton, I. M., Orton, H. D., Randall, C. J., Sam, A., Shore, J.H., Simpson, S.G., Yazzie, L. L., \& AI-SUPERPFP Team. (2003). Racial disparities in alcohol use: Comparison of 2 American Indian reservation populations with national data. American Journal of Public Health, 93, 1683-1685.

Blanchard, K. A., Morgenstern, J., Morgan, T. J., Lobouvie, E. W., \& Bux, D. A. (2003). Assessing consequences of substance Use: Psychometric properties of the Inventory of Drug 
Use Consequences. Psychology of Addictive Behaviors, 17(4), 328-

331. https://doi.org/10.1037/0893-164X.17.4.328

Brave Heart, M. Y. H., Lewis-Fernndez, R., Beals, J., Hasin, D. S., Sugaya, L., Wang, S., Grant, B. F., \& Blanco, C. (2016). Psychiatric disorders and mental health treatment in American Indians and Alaska Natives: Results of the national epidemiologic survey on alcohol and related conditions. Social Psychiatry and Psychiatric Epidemiology, 51, 1033-1046. https://doi.org/10.1007/s00127-016-1225-4

Breslin, F. C., Sobell, L. C., Sobell, M. B., \& Agrawal, S. (2000). A comparison of a brief and long version of the situational confidence questionnaire. Behaviour Research and Therapy, 38, 1211-1220. https://doi.org/10.1016/s0005-7967(99)00152-7

Castor, M. L., Smyser, M. S., Taualii, M. M., Park, A. N., Lawson, S. A., \& Forquera, R. A. (2006). A nationwide population-based study identifying health disparities between American Indians/Alaska Natives and the general populations living in select urban counties. American Journal of Public Health, 96, 1478-1484.

https://doi.org/10.2105/AJPH.2004.053942

Cheadle, J. E., \& Whitbeck, L. B. (2011). Alcohol use trajectories and problem drinking over the course of adolescence: A study of north American indigenous youth and their caretakers. Journal of Health and Social Behavior, 52, 228-245. https://doi.org/10.1177/0022146510393973

Cohen, J. (1988). Statistical power analysis for the behavioral sciences (2nd ed.). Lawrence Erlbaum Associates.

Collins, S. E. (2016). Associations between socioeconomic factors and alcohol outcomes. Alcohol Research: Current Reviews, 38, 83-94. 
Cunningham, J. K., Solomon, T. A., \& Muramoto, M. L. (2016). Alcohol use among Native Americans compared to whites: Examining the veracity of the 'Native American elevated alcohol consumption' belief. Drug and Alcohol Dependence, 160, 65-75. https://doi.org/10.1016/j.drugalcdep.2015.12.015

Daisy, F., Thomas, L. R., \& Worley, C. (1998). Alcohol use and harm reduction within the Native community. In G. A. Marlatt, \& G. A. Marlatt (Eds.), Harm reduction: Pragmatic strategies for managing high-risk behaviors (pp. 327-350). New York, NY: Guilford Press.

Dulin, P. L., Alvarado, C. E., Fitterling, J. M., \& Gonzalez, V. M. (2017). Comparisons of alcohol consumption by timeline follow back vs. smartphone-based daily interviews. Addiction Research \& Theory, 25, 195-200.

https://doi.org/10.1080/16066359.2016.1239081

Ehlers, C. L., \& Gizer, I. R. (2013). Evidence for a genetic component for substance dependence in Native Americans. The American Journal of Psychiatry, 170, 154-164. https://doi.org/10.1176/appi.ajp.2012.12010113 [doi]

Enoch, M., \& Albaugh, B. J. (2017). Review: Genetic and environmental risk factors for alcohol use disorders in American Indians and Alaskan Natives. American Journal on Addictions, 26, 461-468. https://doi.org/10.1111/ajad.12420

Flannery, B. A., Volpicelli, J. R., \& Pettinati, H. M. (1999). Psychometric properties of the Penn Alcohol Craving Scale. Alcoholism: Clinical and Experimental Research, 23, 1289-1295. https://doi.org/10.1111/j.1530-0277.1999.tb04349.x

Gonzalez, V. M., Bravo, A. J., Crouch, M. C., \& Protective Strategies Study Team. (2019). Endorsement of the "firewater myth" affects the use of protective behavioral strategies among American Indian and Alaska Native students. Addictive Behaviors, 93, 78-85. 
https://doi.org/10.1016/j.addbeh.2019.01.027

Gonzalez, V. M., Burroughs, A., \& Skewes M.C. (2021). Belief in the American Indian/Alaska Native biological vulnerability myth and drinking to cope: Does stereotype threat play a role? Cultural Diversity and Ethnic Minority Psychology, 27(1), 37-46. https://doi.org/ $10.1037 / \mathrm{cdp} 0000366$

Gonzalez, V. M., \& Skewes, M. C. (2016). Association of the firewater myth with drinking behavior among American Indian and Alaska Native college students. Psychology of Addictive Behaviors, 30, 838-849. https://doi.org/10.1037/adb0000226

Gonzalez, V. M., \& Skewes, M. C. (2018). Association of belief in the "firewater myth" with strategies to avoid alcohol consequences among American Indian and Alaska Native college students who drink. Psychology of Addictive Behaviors, 32, 401-409. https://doi.org/10.1037/adb0000367

Gordon, S. M., Sterling, R., Siatkowski, C., Raively, K., Weinstein, S., \& Hill, P. C. (2006). Inpatient desire to drink as a predictor of relapse to alcohol use following treatment. American Journal on Addictions, 15, 242-245. https://doi.org/10.1080/10550490600626556 Grant, B. F., Goldstein, R. B., Saha, T. D., Chou, S. P., Jung, J., Zhang, H., Pickering, R. P., Ruan, W. J., Smith, S. M., Huang, B., \& Hasin, D. S. (2015). Epidemiology of DSM-5 alcohol use disorder: Results from the national epidemiologic survey on alcohol and related conditions III. JAMA Psychiatry, 72, 757-766. https://doi.org/10.1001/jamapsychiatry.2015.0584

Greenfield, S. F., Hufford, M. R., Vagge, L. M., Muenz, L. R., Costello, M. E., \& Weiss, R. D. (2000). The relationship of self-efficacy expectancies to relapse among alcohol dependent men and women: A prospective study. Journal of Studies on Alcohol, 61, 345-351. 
https://doi.org/10.15288/jsa.2000.61.345

Hayes, A. F. (2018). Introduction to mediation, moderation, and conditional process analysis (Second edition ed.). New York ; London: Guilford Press.

Israel, B. A., Schulz, A. J., Parker, E. A., \& Becker, A. B. (2008). Critical issues in developing and following community-based participatory research principles. In M. Minkler, \& N. Wallerstein (Eds.), Community-based participatory research for health (pp. 47-62). San Francisco: Jossey-Bass.

Johnson, H. R. (2016). Firewater: How alcohol is killing my people (and yours). University of Regina Press.

Kadden, R. M., \& Litt, M. D. (2011). The role of self-efficacy in the treatment of substance use disorders. Addictive Behaviors, 36, 1120-1126. https://doi.org/10.1016/j.addbeh.2011.07.032

LaMarr, C. J. (2003). "Firewater myth": Fact, fantasy or self-fulfilling prophecy (doctoral dissertation). ProQuest Dissertations Publishing). Retrieved from https://search.proquest.com/docview/305277338

O'Laughlin, K. D., Martin, M.J., \& Ferrer, E. (2018). Cross-sectional analysis of longitudinal mediation processes. Multivariate Behavioral Research, 53(3), 375-402. doi: 10.1080/00273171.2018.1454822.

Miller, W. R., Tonigan, J.S. \& Longabaugh, R. (1995). The Drinker Inventory of Consequences (DrInC): An instrument for assessing adverse consequences of alcohol abuse. Project MATCH Monograph Series, Vol. 4. DHHS Publication No. 95-3911. Rockville, MD: National Institute on Alcohol Abuse and Alcoholism.

O'Connor, P. G., Gottlieb, L. D., Kraus, M. L., Segal, S. R., \& Horwitz, R. I. (1991). Social and 
clinical features as predictors of outcome in outpatient alcohol withdrawal. Journal of General Internal Medicine, 6, 312-316. https://doi.org/10.1007/BF02597427

Osborne, J. W. (2019). Best practices in data cleaning.

Pescosolido, B. A., Martin, J. K., Long, J. S., Medina, T. R., Phelan, J. C., \& Link, B. G. (2010). "A disease like any other"? A decade of change in public reactions to schizophrenia, depression, and alcohol dependence. The American Journal of Psychiatry, 167, 1321-1330. https://doi.org/10.1176/appi.ajp.2010.09121743

Robinson, S. M., Sobell, L. C., Sobell, M. B., \& Leo, G. I. (2014). Reliability of the Timeline Followback for cocaine, cannabis, and cigarette use. Psychology of Addictive Behaviors, 28, 154-162. https://doi.org/10.1037/a0030992

Skewes, M. C., \& Blume, A. W. (2019). Understanding the link between racial trauma and substance use among American Indians. The American Psychologist, 74, 88-100. https://doi.org/10.1037/amp0000331

Skewes, M.C., Gonzalez, V.M., Gameon, J.A., FireMoon, P., Salois, E., Rasmus, S.M., Lewis, J.P., Gardner, S.A., Ricker, A., \& Reum, M. (2020). Health disparities research with American Indian communities: The importance of trust and transparency. American Journal of Community Psychology, https://doi.org/10.1002/ajcp.12445

Skewes, M. C., Hallum-Montes, R., Gardner, S. A., Blume, A. W., Ricker, A., \& FireMoon, P. (2019). Partnering with Native communities to develop a culturally grounded intervention for substance use disorder. American Journal of Community Psychology, 64, 72-82. https://doi.org/10.1002/ajcp.12354

Skewes, M. C., \& Lewis, J. P. (2016). Sobriety and alcohol use among rural Alaska Native elders. International Journal of Circumpolar Health, 75, 1-8. 
Sobell L.C., \& Sobell M.B. (1992) Timeline Follow-Back: A technique for assessing selfreported alcohol consumption (pp. 41-72). In R.Z. Litten \& J.P. Allen (Eds.), Measuring Alcohol Consumption. Humana Press. https://doi.org/10.1007/978-1-4612-0357-5_3

Sobell, L. C., Sobell, M. B., Connors, G. J., \& Agrawal, S. (2003). Assessing drinking outcomes in alcohol treatment efficacy studies: Selecting a yardstick of success. Alcoholism: Clinical and Experimental Research, 27, 1661-1666. https://doi.org/10.1097/01.ALC.0000091227.26627.75

Sobell, M. B., Sobell, L. C., Bogardis, J., \& Leo, G. I. (1992). Problem drinkers' perceptions of whether treatment goals should be self-selected or therapist-selected. Behavior Therapy, 23, 43-52. https://doi.org/10.1016/S0005-7894(05)80307-7

Stohs, M. E., Schneekloth, T. D., Geske, J. R., Biernacka, J. M., \& Karpyak, V. M. (2019). Alcohol craving predicts relapse after residential addiction treatment. Alcohol and Alcoholism, 54(2), 167-172. https://doi.org/10.1093/alcalc/agy093

Tabachnick, B. G., \& Fidell, L. S. (2014). Using multivariate statistics (6th ed.). Pearson. Thatcher, R. W. (2004). Fighting firewater fictions: Moving beyond the disease model of alcoholism in First Nations. University of Toronto Press.

Tonigan, J. S., \& Miller, W. R. (2002). The Inventory of Drug Use Consequences (InDUC): Test-retest stability and sensitivity to detect change. Psychology of Addictive Behaviors, 16(2), 165-168. https://doi.org/10.1037/0893-164X.16.2.165

Vaeth, P. A., Wang-Schweig, M., \& Caetano, R. (2017). Drinking, alcohol use disorder, and treatment access and utilization among U.S. racial/ethnic groups. Alcoholism, Clinical and Experimental Research, 41, 6-19. https://doi.org/10.1111/acer.13285 [doi]

Wallerstein, N. B., \& Duran, B. (2010). Community-based participatory research contributions to 
intervention research: The intersection of science and practice to improve health equity. American Journal of Public Health, 100, S40-S46.

https://doi.org/10.2105/ajph.2009.184036

Wallerstein, N. B., \& Duran, B. (2016). Using community-based participatory research to address health disparities. Health Promotion Practice, 7, 312-323.

https://doi.org/10.1177/1524839906289376

Whitbeck, L. B., Chen, X., Hoyt, D. R., \& Adams, G. W. (2004). Discrimination, historical loss and enculturation: Culturally specific risk and resiliency factors for alcohol abuse among American Indians. Journal of Studies on Alcohol, 65, 409-418.

https://doi.org/10.15288/jsa.2004.65.409

Whitbeck, L. B., Hoyt, D. R., McMorris, B. J., Chen, X., \& Stubben, J. D. (2001). Perceived discrimination and early substance abuse among American Indian children. Journal of Health and Social Behavior, 42, 405-424. https://doi.org/10.2307/3090187

Whitesell, N. R., Beals, J., Crow, C. B., Mitchell, C. M., \& Novins, D. K. (2012). Epidemiology and etiology of substance use among American Indians and Alaska Natives: Risk, protection, and implications for prevention. The American Journal of Drug and Alcohol Abuse, 38, 376-382. https://doi.org/10.3109/00952990.2012.694527 


\section{Table 1}

Participant Characteristics $(N=141)$

\begin{tabular}{|c|c|c|}
\hline Characteristics & $n$ & $\%$ \\
\hline \multicolumn{3}{|l|}{ Gender } \\
\hline Female & 67 & 47.5 \\
\hline Male & 74 & 52.5 \\
\hline \multicolumn{3}{|l|}{ Education, $n(\%)$} \\
\hline Some college/college degree & 14 & 9.9 \\
\hline High school graduate/GED & 38 & 27.0 \\
\hline Some high school & 66 & 46.8 \\
\hline Middle school (7-8 grade) & 12 & 8.5 \\
\hline Not reported & 11 & 7.8 \\
\hline \multicolumn{3}{|l|}{ Monthly income } \\
\hline$>\$ 2,000$ & 7 & 5.0 \\
\hline$\$ 1,000-\$ 2,000$ & 9 & 6.4 \\
\hline$\$ 500-\$ 1,000$ & 19 & 13.5 \\
\hline$\$ 1-500$ & 22 & 15.6 \\
\hline$\$ 0$ & 55 & 39.0 \\
\hline Not reported & 29 & 20.6 \\
\hline \multicolumn{3}{|l|}{ Time in recovery } \\
\hline Over a year & 43 & 30.5 \\
\hline Less than a year & 47 & 33.3 \\
\hline Less than a month & 17 & 12.1 \\
\hline Not yet in recovery or just beginning & 25 & 17.7 \\
\hline Not reported & 9 & 6.4 \\
\hline Previous substance use treatment $\mathrm{t}^{\mathrm{a}}$ & 92 & 65.2 \\
\hline Cannabis use ${ }^{\mathrm{b}}$ & 59 & 41.8 \\
\hline
\end{tabular}

${ }^{a}$ Previous substance use treatment included formal treatment $(n=77)$, Alcoholics Anonymous $(n$ $=73)$, and/or Narcotics Anonymous $(n=28)$.

${ }^{b}$ No significant difference was found in cannabis use between those who used alcohol only and those who reported both alcohol and hard drug use $\left(\chi^{2}=3.21, p=.073\right)$. 


\section{$1 \quad$ Table 2}

2 Bivariate Correlations Among Study Variables

\begin{tabular}{|c|c|c|c|c|c|c|c|c|c|c|c|}
\hline & 1 & 2 & 3 & 4 & 5 & 6 & 7 & 8 & $M$ & $S D$ & Range \\
\hline $\begin{array}{l}\text { 1. Belief in a biological } \\
\text { vulnerability }\end{array}$ & - & & & & & & & & 3.86 & 1.58 & $1-6$ \\
\hline 2. Self-efficacy & .07 & - & & & & & & & 52.69 & 20.73 & $1-100$ \\
\hline 3. Craving & .16 & $-.40^{* * * *}$ & - & & & & & & 16.76 & 7.53 & $5-34$ \\
\hline 4. Drinking days ${ }^{\mathrm{a}}$ & .08 & $-.34^{* * *}$ & $.39^{* * *}$ & - & & & & & 26.57 & 28.26 & $1-90$ \\
\hline 5. Drinks per drinking day & -.03 & -.05 & .06 & .10 & - & & & & 17.97 & 13.16 & $1-60$ \\
\hline $\begin{array}{l}\text { 6. Substance use } \\
\text { consequences }^{\mathrm{a}}\end{array}$ & .09 & $-.39^{* * *}$ & $.46^{* * *}$ & $.37^{* * *}$ & .12 & - & & & 17.57 & 14.95 & $0-50$ \\
\hline 7. Substance use group ${ }^{b}$ & -.09 & $-.18^{*}$ & $.21^{*}$ & .04 & .06 & $.25^{* *}$ & - & & - & - & - \\
\hline 8. Age & .09 & -.14 & $.25^{* *}$ & $.28^{* * *}$ & -.09 & .16 & $-.18^{*}$ & - & 37.98 & 11.76 & $18-63$ \\
\hline 9. Gender ${ }^{\mathrm{c}}$ & -.09 & -.02 & -.03 & -.14 & $-.28^{* * *}$ & .03 & .08 & .03 & - & - & - \\
\hline
\end{tabular}




\section{Table 3}

9 Alcohol Use as a Mediator of the Association of Belief in an AIAN Specific Biological

Vulnerability (BV) with Substance Use Consequences

\begin{tabular}{|c|c|c|c|c|c|c|}
\hline \multirow[t]{2}{*}{ Belief in BV $\rightarrow$ alcohol use models } & \multicolumn{3}{|c|}{$\begin{array}{c}\text { Drinking days } \\
\left(R^{2}=.18\right)\end{array}$} & \multicolumn{3}{|c|}{$\begin{array}{l}\text { Drinks per drinking day } \\
\qquad\left(R^{2}=.10\right)\end{array}$} \\
\hline & $\mathrm{B}(S E)$ & $\beta$ & $f^{2}$ & $\mathrm{~B}(S E)$ & $\beta$ & $f^{2}$ \\
\hline Belief in BV & $0.42(0.17)$ & $.25^{*}$ & .05 & $-0.02(0.10)$ & -.02 & .00 \\
\hline Substance use group & $0.59(0.43)$ & .23 & .01 & $0.19(0.25)$ & .13 & .00 \\
\hline $\mathrm{BV} \times$ Substance use group & $-0.86(0.27)$ & $-.51 * *$ & .07 & $-0.06(0.16)$ & -.07 & .00 \\
\hline \multicolumn{7}{|l|}{ Covariates } \\
\hline Age & $0.07(0.02)$ & $.30 * * *$ & .11 & $-0.01(0.01)$ & -.07 & .00 \\
\hline Gender & $-1.03(0.42)$ & $-.39 *$ & .04 & $-0.86(0.24)$ & $-.59 * *$ & .09 \\
\hline \multirow[t]{2}{*}{ Alcohol use $\rightarrow$ consequence models } & \multicolumn{3}{|c|}{ Consequences $\left(R^{2}=.21\right)$} & \multicolumn{3}{|c|}{ Consequences $\left(R^{2}=.14\right)$} \\
\hline & $\mathrm{B}(S E)$ & $\beta$ & $f^{2}$ & $\mathrm{~B}(S E)$ & $\beta$ & $f^{2}$ \\
\hline Drinking days & $.23(0.09)$ & $.29 *$ & .05 & - & - & - \\
\hline Drinks/day & - & - & - & $0.24(0.17)$ & .16 & .01 \\
\hline Belief in BV & $0.15(0.14)$ & .11 & .01 & $0.25(0.14)$ & .18 & .02 \\
\hline Substance use group & $1.12(0.34)$ & $.52 * *$ & .08 & $1.23(0.36)$ & $.57 * *$ & .09 \\
\hline $\begin{array}{l}\text { Drinking days } \times \text { Substance use } \\
\text { group }\end{array}$ & $0.07(0.13)$ & .08 & .00 & - & - & - \\
\hline Drinks/day $\times$ Substance use group & - & - & - & $-0.08(0.24)-$ & -.05 & .00 \\
\hline BV $\times$ Substance use group & $-0.05(0.22)$ & -.04 & .00 & $-0.26(0.23)$ & -.19 & .01 \\
\hline \multicolumn{7}{|l|}{ Covariates } \\
\hline Age & $0.02(0.02)$ & .13 & .01 & $0.04(0.01)$ & $.22 * *$ & .05 \\
\hline Gender & $0.22(0.34)$ & .11 & .00 & $0.12(0.37)$ & .06 & .00 \\
\hline
\end{tabular}

11 Note. Substance use group was coded: alcohol use-only group=0 and alcohol and hard drug use

12 group $=1$. Gender was coded: $m e n=0$ and women=1. Substance use consequences, drinking days,

13 and drinks per drinking day were square-root transformed prior to analyses. An effect size $\left(f^{2}\right)$ of

14.02 is conventionally interpreted as small, .15 as moderate, and .35 as large (Cohen, 1988).

$15 * p<.05 . * * p<.01 . * * * p<.001$. 
Table 4

17 Self-Efficacy as a Mediator of the Association of Belief in an AIAN Specific Biological

Vulnerability (BV) with Drinking Days and Drinks Per Drinking Day

\begin{tabular}{|c|c|c|c|c|c|c|}
\hline \multirow[t]{2}{*}{ Belief in BV $\rightarrow$ self-efficacy model } & \multicolumn{6}{|c|}{$\begin{array}{c}\text { Self-efficacy } \\
\left(R^{2}=.07\right)\end{array}$} \\
\hline & \multicolumn{2}{|c|}{$\mathrm{B}(S E)$} & $\beta$ & \multicolumn{2}{|l|}{$f^{2}$} & \\
\hline Belief in BV & \multicolumn{2}{|c|}{$0.80(1.43)$} & .06 & .00 & & \\
\hline Substance use group & \multicolumn{2}{|c|}{$-.8 .75(3.58)$} & $-.42 *$ & .04 & & \\
\hline $\mathrm{BV} \times$ Substance use group & \multicolumn{2}{|c|}{$0.13(2.26)$} & .01 & .00 & & \\
\hline \multicolumn{7}{|l|}{ Covariates } \\
\hline Age & \multicolumn{2}{|c|}{$-0.32(0.15)$} & $-.18^{*}$ & .03 & & \\
\hline Gender & \multicolumn{2}{|c|}{$0.36(3.51)$} & .02 & .00 & & \\
\hline \multirow[t]{2}{*}{ Alcohol use $\rightarrow$ consequence models } & \multicolumn{3}{|c|}{$\begin{array}{l}\text { Drinking days } \\
\quad\left(R^{2}=.26\right)\end{array}$} & \multicolumn{3}{|c|}{$\begin{array}{l}\text { Drinks per drinking day } \\
\qquad\left(R^{2}=.10\right)\end{array}$} \\
\hline & $\mathrm{B}(S E)$ & $\beta$ & $f^{2}$ & $\mathrm{~B}(S E)$ & $\beta$ & $f^{2}$ \\
\hline Self-efficacy & $-0.04(0.01)$ & $-.31 * *$ & .07 & $-0.01(0.01)$ & -.08 & .00 \\
\hline Belief in BV & $0.45(0.16)$ & $.27 * *$ & .06 & $-0.01(0.10)$ & -.02 & .00 \\
\hline Substance use group & $0.25(0.42)$ & .10 & .00 & $0.16(0.25)$ & .11 & .00 \\
\hline Self-efficacy $\times$ Substance use group & $0.00(0.02)$ & .00 & .00 & $0.00(0.01)$ & .05 & .00 \\
\hline BV $\times$ Substance use group & $-0.86(0.26)$ & $-.51 * *$ & .08 & $-0.06(.16)$ & -.07 & .00 \\
\hline \multicolumn{7}{|l|}{ Covariates } \\
\hline Age & $0.05(0.02)$ & $.24 * *$ & .07 & $-0.01(0.01)$ & -.08 & .01 \\
\hline Gender & $-1.02(0.40)$ & $-.38 *$ & .05 & $-0.86(0.24)$ & $-.59 * *$ & .09 \\
\hline
\end{tabular}

Note. Substance use group was coded: alcohol use-only group $=0$ and alcohol and hard drug use

20 group $=1$. Gender was coded: $m e n=0$ and women=1. Drinking days and drinks per drinking day

21 were square-root transformed prior to analyses.

$22 * p<.05 . * * p<.01 . * * * p<.001$. 


\section{$24 \quad$ Table 5}

25 Craving as a Mediator of the Association of Belief in an AIAN Specific Biological Vulnerability

26 (BV) with Drinking Days and Drinks Per Drinking Day

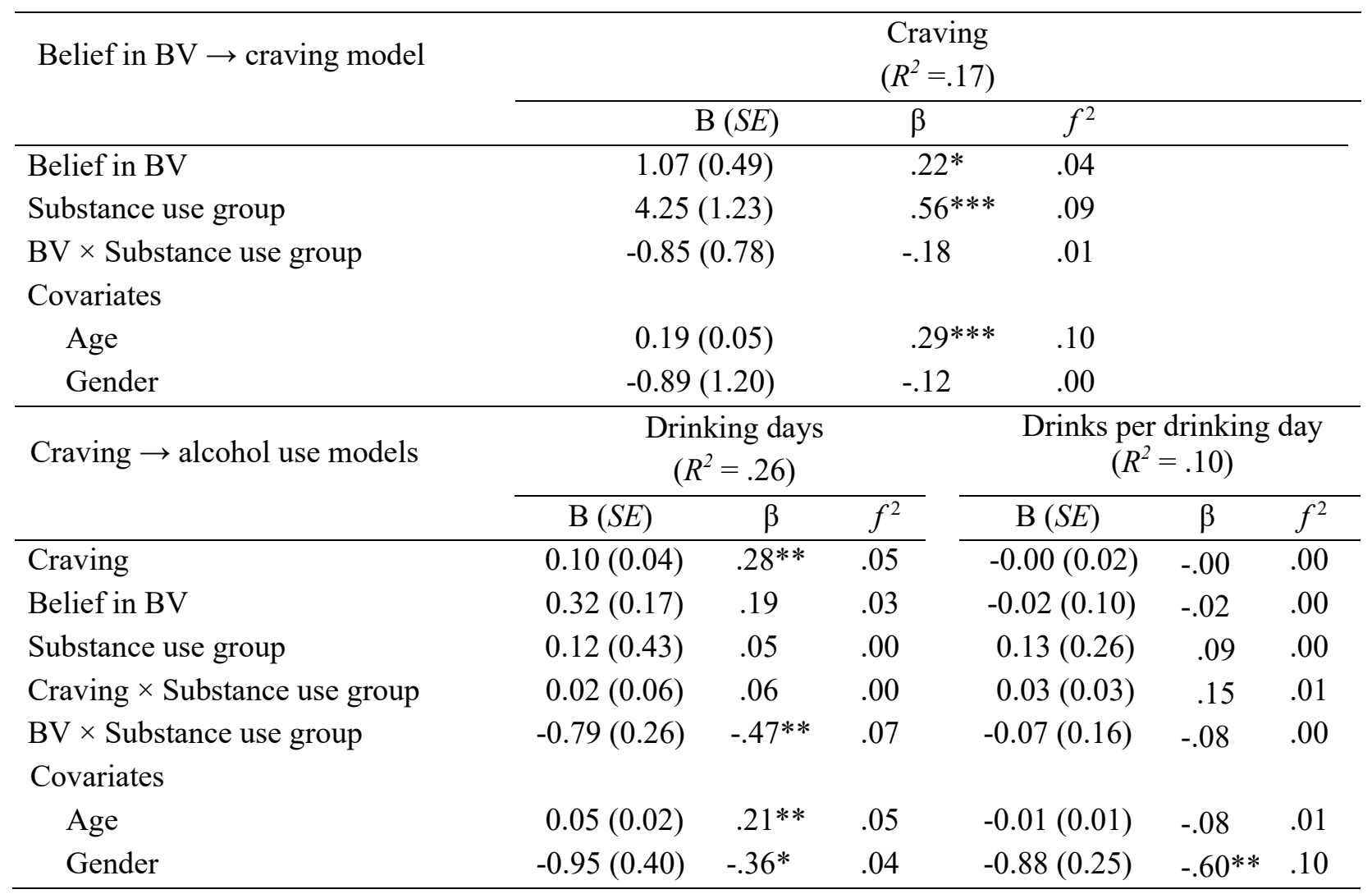

27 Note. Substance use group was coded: alcohol use-only group=0 and alcohol and hard drug use

28 group $=1$. Gender was coded: $m e n=0$ and women=1. Drinking days and drinks per drinking day 29 were square-root transformed prior to analyses.

$30 * p<.05 . * * p<.01 . * * * p<.001$. 


\section{$32 \quad$ Figure 1}

33 Moderated-Mediation Models of Belief in an AIAN Biological Vulnerability with Substance Use

34 Consequences and Drinking Days

35

36
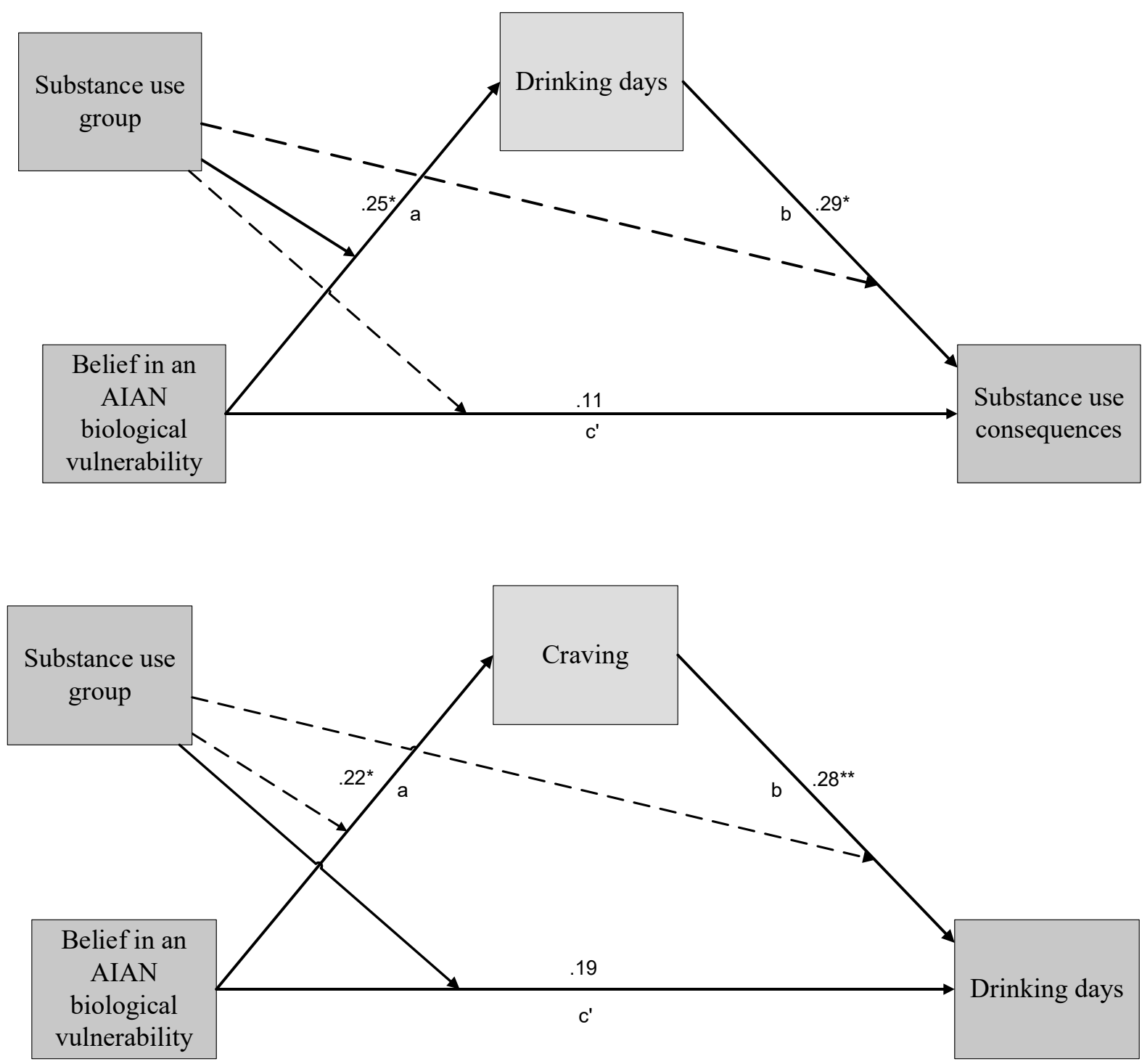

38 Note. Depicted above are the standardized regression coefficients for model paths. Significant interactions for substance use group are depicted above by a solid line pointing to the given path; dashed lines indicate statistical significance was not found. Gender and age were included as

41 covariates.

$42 * p<.05 * * p<.01$. 


\section{$44 \quad$ Figure 2}

45 Relationship of Belief in an AIAN Biological Vulnerability with Drinking Days as Moderated by

$46 \quad$ Substance Use Group

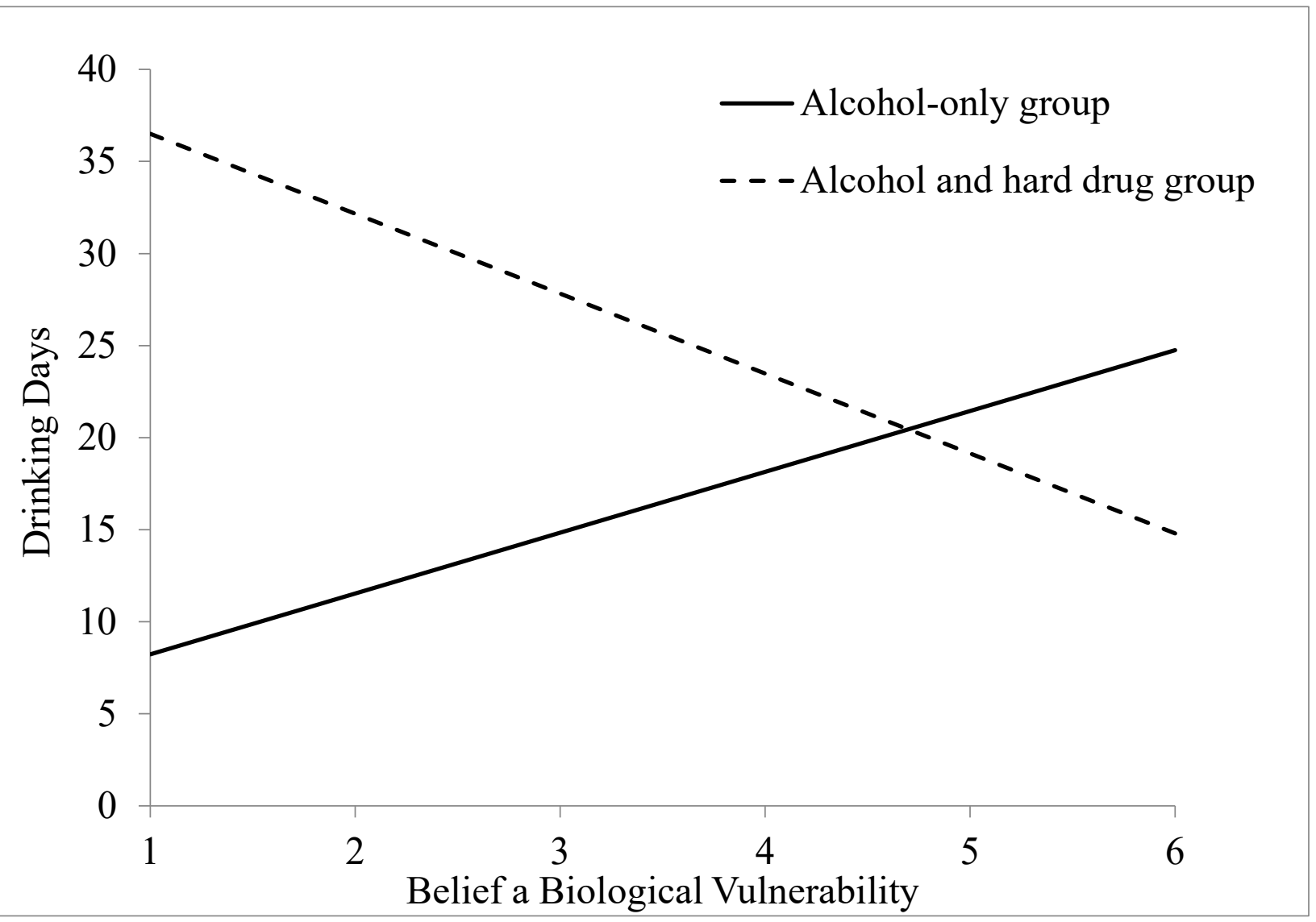

48 Note. For the analyses drinking days was square-root transformed. To aid in interpretation,

49 drinking days $\left(Y^{\prime}\right)$ was backtransformed for the figure.

50

51 